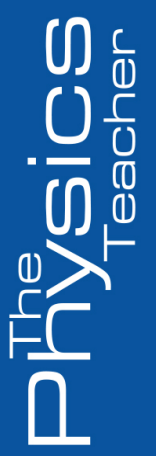

\title{
Apparatus for rotational motion
}

Cite as: The Physics Teacher 33, 173 (1995); https://doi.org/10.1119/1.2344182

Published Online: 04 June 1998

\section{Said Shakerin}

\section{ARTICLES YOU MAY BE INTERESTED IN}

Vortex Apparatus and Demonstrations

The Physics Teacher 48, 316 (2010); https://doi.org/10.1119/1.3393063

\section{Angle of repose}

The Physics Teacher 54, 192 (2016); https://doi.org/10.1119/1.4942158

Fluids Demonstrations II: Bubbles in Mondrian Painting, Eruption-Like Flow, Rotational Instability, and Wake Vortices

The Physics Teacher 57, 600 (2019); https://doi.org/10.1119/1.5135786

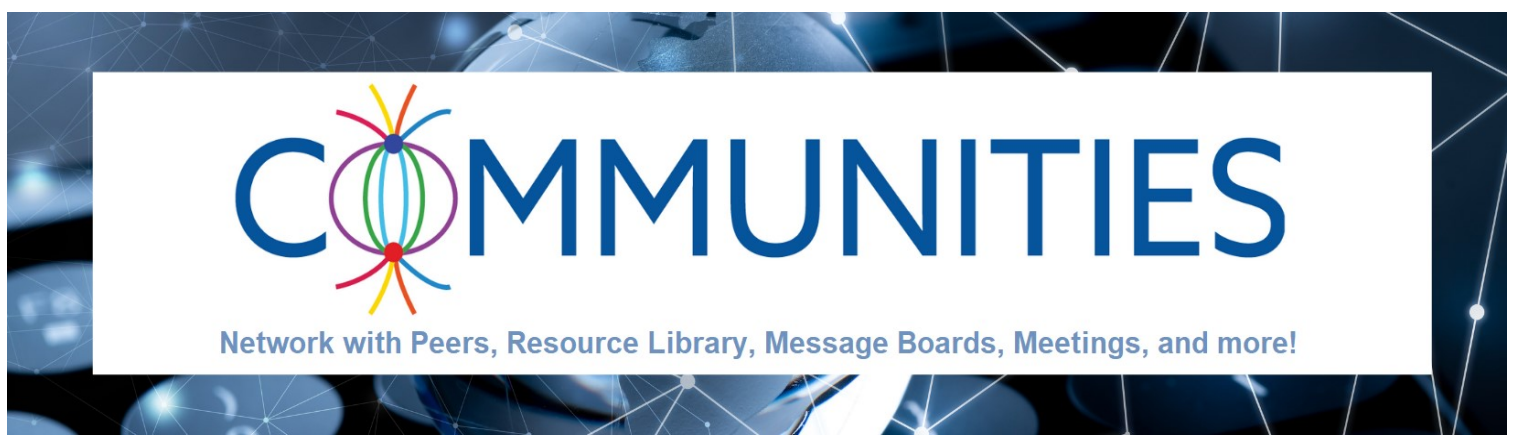


complete and the helium allowed to escape. The volume of the balloon may be found either by displacement of water or by approximating the shape of a disk or an ellipsoid (Vellipsoid $=4 / 3 \pi a^{2} b$ ).

\section{Data}

Typical data for this experiment:

$$
\begin{aligned}
& a=17.5 \mathrm{~cm} \\
& b=8.5 \mathrm{~cm} \\
& V=11,000 \mathrm{~cm}^{3}
\end{aligned}
$$

$$
\begin{aligned}
& \rho_{A}=1.20 \times 10^{-3} \mathrm{~g} / \mathrm{cm}^{3} \\
& \rho_{H e}=0.18 \times 10^{-3} \mathrm{~g} / \mathrm{cm}^{3}
\end{aligned}
$$

Mass of gondola and balloon $=12.60 \mathrm{~g}$.

Our measurements for the buoyant force agreed with our calculations with a discrepancy of about $10 \%$.

\section{Reference}

1. Handbook of Chemistry and Physics, 45th edition, edited by R.C. Weast (The Chemical Rubber Company, Cleveland, $\mathrm{OH}, 1964)$, pp. B-178 and F-8.

\title{
Apparatus for Rotational Motion
}

\author{
Said Shakerin, Department of Mechanical Engineering, \\ University of the Pacific, Stockton, CA 95211
}

A simple device for demonstration of rotational motion is shown in Fig. 1. Place a wooden bead on a $20-\mathrm{cm}$ circular loop of copper wire (diameter 0.4 $\mathrm{cm}$ ) which is brazed to a straight $5-\mathrm{cm}$ handle. Insert the handle in a hand drill. As the drill starts to rotate, while the handle is oriented vertically, the bead

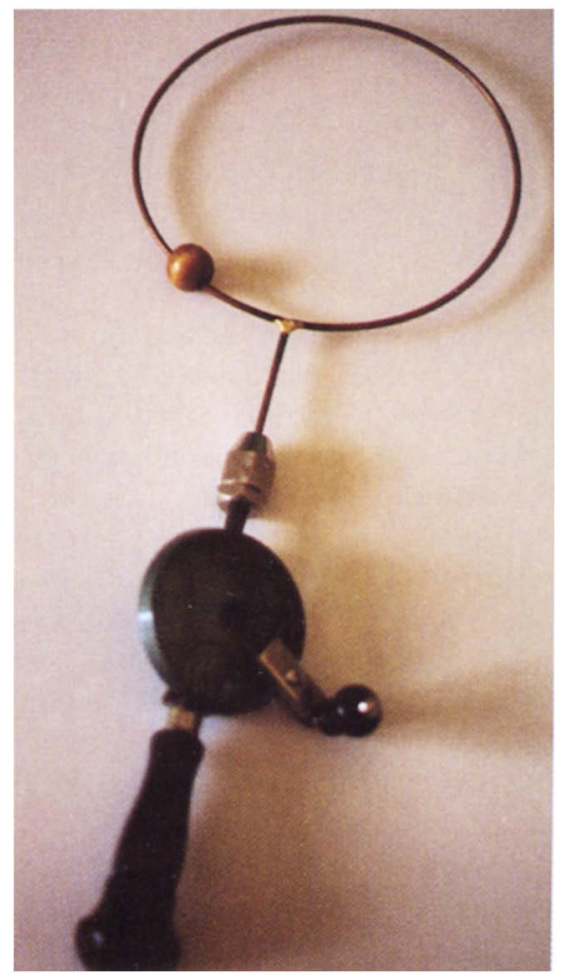

Flg. 1. Teaching tool for rotational motion. moves up from the bottom position. The position of the bead on the loop depends on the angular velocity of the drill. Regardless of this velocity, however, the bead never crosses the horizontal midpoint on the loop. Relevant analysis follows.

Assuming the bead is free to move along the loop (i.e., negligible friction between the loop and the bead), the equations of motion along the radial and vertical directions are:
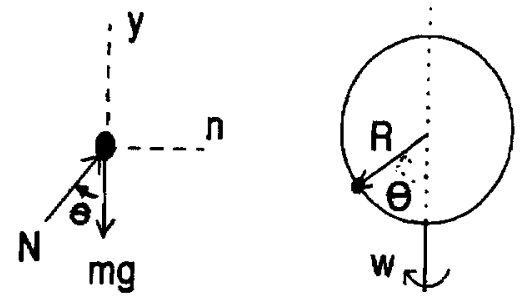

Fig. 2.

$$
\begin{gathered}
\Sigma F_{n}=m a_{n} \rightarrow N \sin \theta=R w^{2} \sin \theta \\
\Sigma F_{y}=0 \rightarrow m g-N \cos \theta=0
\end{gathered}
$$

Combining the above equations and solving for $w$, yields

$$
w=\left(\frac{g}{R \cos \theta}\right)^{\frac{1}{2}}
$$

Note: at $\theta=90^{\circ}, w \rightarrow \infty$

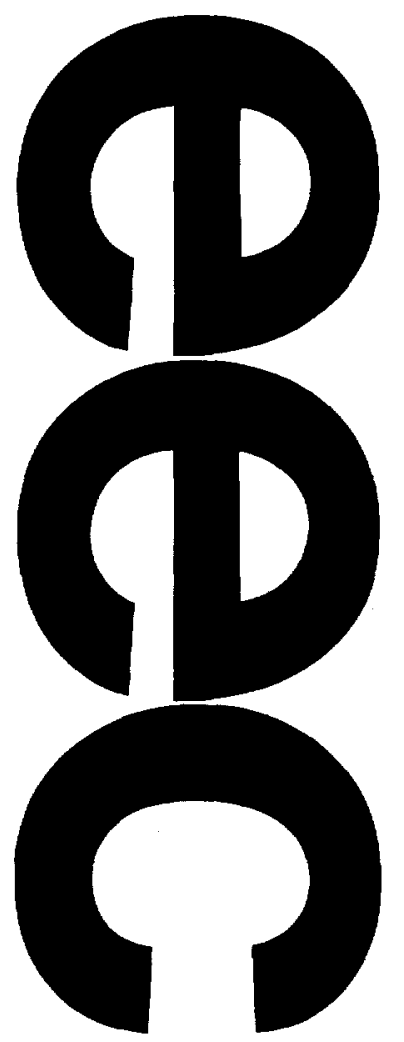

Your one source for physics equipment that is:

- colorful

- reliable

- easy to use

- straightforward

- thoroughly documented

- unquestionably guaranteed and comes with a 30-day free trial.

Write for a free catalog of physics equipment you've never seen before.

\section{eec}

EDUCATIONAL EQUIPMENT COMPANY PO BOX 2102

VERNON, CT 06066-2102 USA

(203) 647-0061 